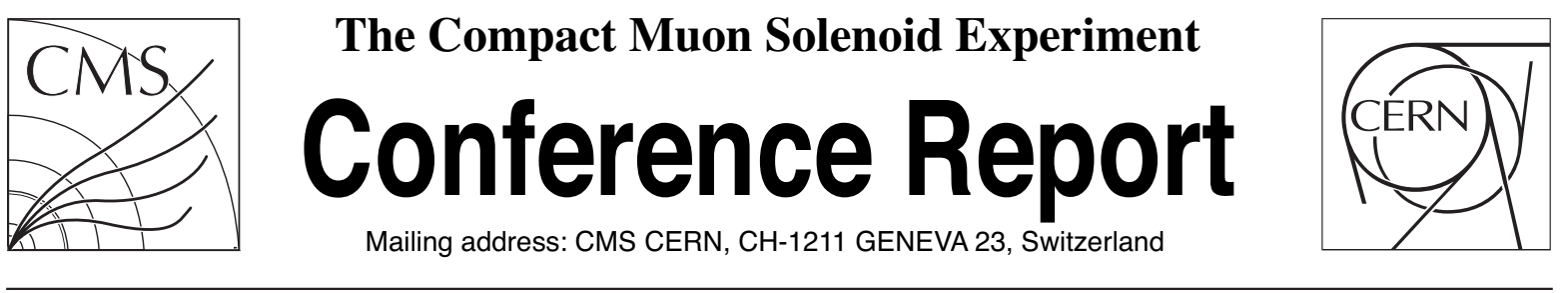

30 March 2014 (v2, 01 April 2014)

\title{
Jet shape and fragmentation function measurements with CMS
}

\author{
Yaxian Mao for the CMS Collaboration
}

\begin{abstract}
We present measurements of the jet fragmentation functions and transverse momentum profiles (shapes) in $\mathrm{PbPb}$ collisions at $2.76 \mathrm{TeV}$ per nucleon-pair center of mass energy with the CMS detector. Jets are reconstructed by the anti- $k_{T}$ sequential clustering algorithm with a resolution parameter of 0.3 , using particle-flow objects that combine tracking and calorimetric information. In this talk the jet properties of inclusive jets with transverse momentum $p_{T}>100 \mathrm{GeV} / \mathrm{c}$ in $\mathrm{PbPb}$ collisions are measured for reconstructed charged particles with $p_{T}>1 \mathrm{GeV} / \mathrm{c}$ in a cone of radius $\sqrt{\Delta \phi^{2}+\Delta \eta^{2}}=$ 0.3 around the jet axis. A data sample of collisions collected in 2011 at a center-of-mass energy of $\sqrt{s_{N N}}=2.76 \mathrm{TeV}$ corresponding to an integrated luminosity of $150 \mu b^{-1}$ is used. The results for $\mathrm{PbPb}$ collisions as a function of collision centrality are compared to reference distributions based on the full 2013 pp statistics collected at the same collision energy. Centrality-dependent modifications of the fragmentation functions and shapes are revealed.
\end{abstract}

Presented at HardProbes2013 6th International Conference on Hard and Electromagnetic Probes of High-Energy Nuclear Collisions 


\title{
Jet shape and fragmentation function measurements with CMS
}

\author{
Yaxian MAO (for the CMS Collaboration) ${ }^{1}$ \\ Department of Physics and Astronomy, Vanderbilt University, Nashville, TN37235, USA
}

\begin{abstract}
We present measurements of the jet fragmentation functions and transverse momentum profiles (shapes) in $\mathrm{PbPb}$ collisions at 2.76 $\mathrm{TeV}$ per nucleon-pair center of mass energy with the CMS detector. Jets are reconstructed by the anti- $k_{T}$ sequential clustering algorithm with a resolution parameter of 0.3 , using particle-flow objects that combine tracking and calorimetric information. In this talk the jet properties of inclusive jets with transverse momentum $p_{T}>100 \mathrm{GeV} / \mathrm{c}$ in $\mathrm{PbPb}$ collisions are measured for reconstructed charged particles with $p_{T}>1 \mathrm{GeV} / \mathrm{c}$ in a cone of radius $\sqrt{\Delta \phi^{2}+\Delta \eta^{2}}=0.3$ around the jet axis. A data sample of $\mathrm{PbPb}$ collisions collected in 2011 at a center-of-mass energy of $\sqrt{s_{N N}}=2.76 \mathrm{TeV}$ corresponding to an integrated luminosity of 150 $\mu b^{-1}$ is used. The results for $\mathrm{PbPb}$ collisions as a function of collision centrality are compared to reference distributions based on the full 2013 pp statistics collected at the same collision energy. Centrality-dependent modifications of the fragmentation functions and shapes are revealed.
\end{abstract}

Keywords: jet quenching, jet fragmentation, jet shapes

\section{Introduction}

Hard probes - processes involving scatterings with large momentum transfer - and their modification in $\mathrm{PbPb}$ collisions with respect to pp collisions, can be used to study the transport properties of the hot quark-gluon plasma (QGP) medium created in heavy ion collisions at ultra-relativistic energies [1]. The objective is to understand how a color charge (carried by quarks and gluons) is transported in the colored medium. It is well established that the parton energy loss is a consequence of the modification of the hard-scattering processes in the final state of the heavy ion collisions at RHIC and LHC [2-4]. It is evident in the suppression of high $p_{T}$ charged hadrons and jets, as well as in the associated yield in two-particle correlations measurements [5], which is accompanied by an enhancement of low $p_{T}$ hadrons, suggesting a softening of jet fragmentations. Those observations raise questions related to the parton energy loss mechanism: how do partons lose their energy in the nuclear medium? Does the energy loss change the fragmentation pattern of the jets and modify the jet shape? The CMS experiment at the LHC is ideally suited to measure these hard probes in more detail, due to its large acceptance, good efficiency and low fake rate for track reconstruction, and high level trigger capable of full event reconstruction even in a high multiplicity environment [6]. Taking advantage of the high statistics data collected in 2011, the present analysis enables us to study the parton energy loss in more detail than previously possible. Measurements of jet fragmentation properties provide an experimental constraint which is complementary to the measurement of the global jet properties, and can also be used to connect the jet observables to the measurements of high $p_{T}$ particle production.

\footnotetext{
${ }^{1} \mathrm{~A}$ list of members of the CMS Collaboration and acknowledgements can be found at the end of this issue.
} 
In the following, we discuss the measurements of the jet fragmentation and the jet shapes with CMS using the data collected during 2011 for PbPbcollisions and 2013 for ppreference runs at the same nucleon-nucleon centre-of-mass energy of $\sqrt{s_{N N}}=2.76 \mathrm{TeV}$. For the present analysis, the events with jets above $p_{T}>100 \mathrm{GeV} / c$ within $0.3<|\eta|<2$ region are selected, where the jet trigger is fully efficient.

In heavy-ion collisions, particles from the underlying event that happen to fall inside the jet cone would affect the measurements. This contribution is subtracted following a procedure previously employed by CMS in the measurement of the jet fragmentation function, using a method called the $\eta$-reflection method [7]. Therefore the central pseudorapidity region $|\eta|<0.3$ is excluded to avoid jet cones overlapping with the $\eta$-reflected cone used for the background estimation. The minimum transverse momentum for tracks entering the analysis is set to $1 \mathrm{GeV} / c$.

\section{Measurements of jet shapes}

The jet shapes, describing how the transverse momentum of the particles comprising the jet is distributed radially with respect to the jet axis, are studied to gain more insight on the energy loss mechanism of parton-medium interactions [8]. The differential jet shape, $\rho(r)$, describes the radial distribution of transverse momentum inside the jet cone:

$$
\rho(r)=\frac{1}{\delta r} \frac{1}{N_{\text {jet }}} \sum_{\text {jets }} \frac{\sum_{\text {tracks } \in\left[r_{a}, r_{b}\right)} p_{T}^{\text {track }}}{p_{T}^{\text {jet }}}
$$

where the jet cone is divided into six annuli with radial width $\delta r=0.05$, and each annulus has an inner radius of $r_{\mathrm{a}}=r-\delta r / 2$ and outer radius of $r_{\mathrm{b}}=r+\delta r / 2$. Here $r=\sqrt{\left(\eta_{\text {track }}-\eta_{\text {jet }}\right)^{2}+\left(\phi_{\text {track }}-\phi_{\text {jet }}\right)^{2}} \leq 0.3$ is the reconstructed track's radial distance from the jet axis, defined by the coordinates $\eta_{\text {jet }}$ and $\phi_{\text {jet }}$.

The measured jet shapes in $\mathrm{PbPb}$ collisions are compared with the shapes obtained in pp collisions in the top row of Fig. 1 for five centrality classes, varying from most peripheral 70-100\% (left) to most central 0-10\% (right) [9]. In the peripheral collisions (70-100\%), the ratio is close to unity within the uncertainties in the whole measured range, indicating that the radial distribution of the summed transverse momentum of the particles inside the jets is similar in the two systems. In more central PbPbcollisions (0-70\%), a depletion is observed in the region $0.1<r<0.2$. In the most central $\mathrm{PbPbcollisions} \mathrm{(10-30 \%} \mathrm{and} \mathrm{0-10 \% ),} \mathrm{an} \mathrm{excess} \mathrm{of} \mathrm{transverse} \mathrm{momentum} \mathrm{fraction} \mathrm{emitted} \mathrm{at} \mathrm{large} \mathrm{radius}$ $r>0.2$ emerges, indicating a moderate broadening of the jets in the medium. These observations are consistent with previous studies in CMS which find that the energy that the jets lose in the medium is redistributed at large distances from the jet axis outside the jet cone [10].

\section{Measurements of jet fragmentation functions}

To study how the jet energy redistributed in momentum space, one can measure the jet fragmentation functions by correlating reconstructed charged-particle tracks contained within the jet cones, around the axis of the respective jet. As done in previous measurements [7], the fragmentation function is presented as a function of the track $p_{T}$ and $\xi$, defined as:

$$
\xi=\ln \left(p^{j e t} / p_{\|}^{\text {track }}\right)
$$

where $p_{\|}^{\text {track }}$ is the projection of the track momentum onto the jet axis [11].

The measured jet fragmentation functions show similar centrality dependence when compared to pp reference as observed for jet shape measurements. The ratio of jet fragmentation function $\mathrm{Pbb} / \mathrm{pp}$ is almost flat at unity which means no modification for non-central collisions, while a significant excess at high $\xi$ (left) or low track $p_{T}$ (right) and a suppression at immediate $\xi$ and $p_{T}$ is observed for more central events (Fig. 2). This implies that for central collisions the spectrum of particles in a jet has an enhanced contribution of soft particles compared to that from ppcollisions.

Both jet shape and fragmentation function results show quite similar curves in the momentum space and $\eta-\phi$ plane: a depletion at intermediate range and an enhancement at large radii and $\xi$. Therefore, one can map these corrections by looking at the pure generator level distribution. From PYTHIA [12] generated jet, the distribution of charged particle fraction inside jet cones for various $p_{T}$ bins are shown as a function of the distance from jet axis (Fig. 3), from which one can easily translate one measurement to another. Since intermediate $p_{T}$ particles are mainly 


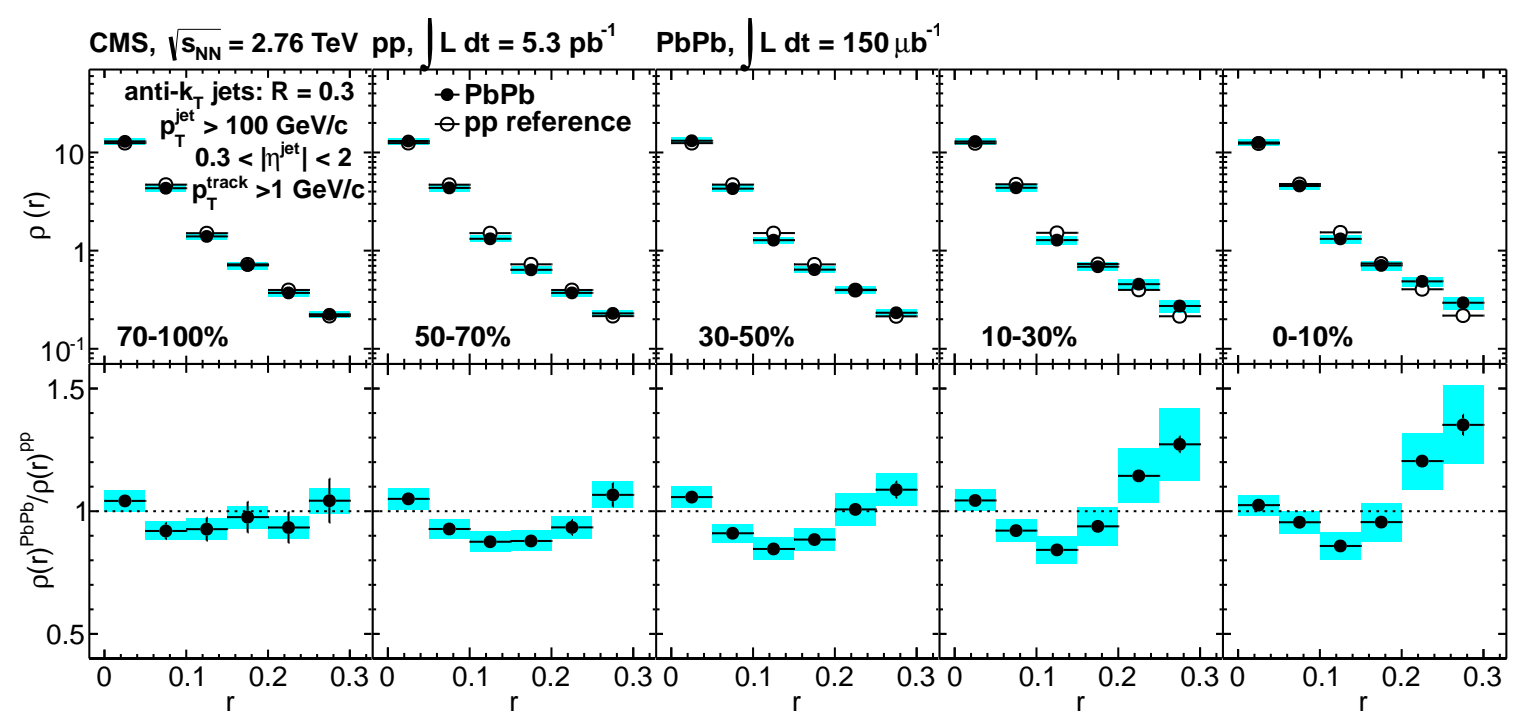

Figure 1. Differential jet shapes in $\mathrm{PbPb}$ and pp collisions for $p_{T}^{\text {jet }}>100 \mathrm{GeV} / c$ with track $p_{T}>1 \mathrm{GeV} / c$ (top panels) and the ratio of the $\mathrm{PbPb}$ and pp jet shapes (bottom panels) [9].
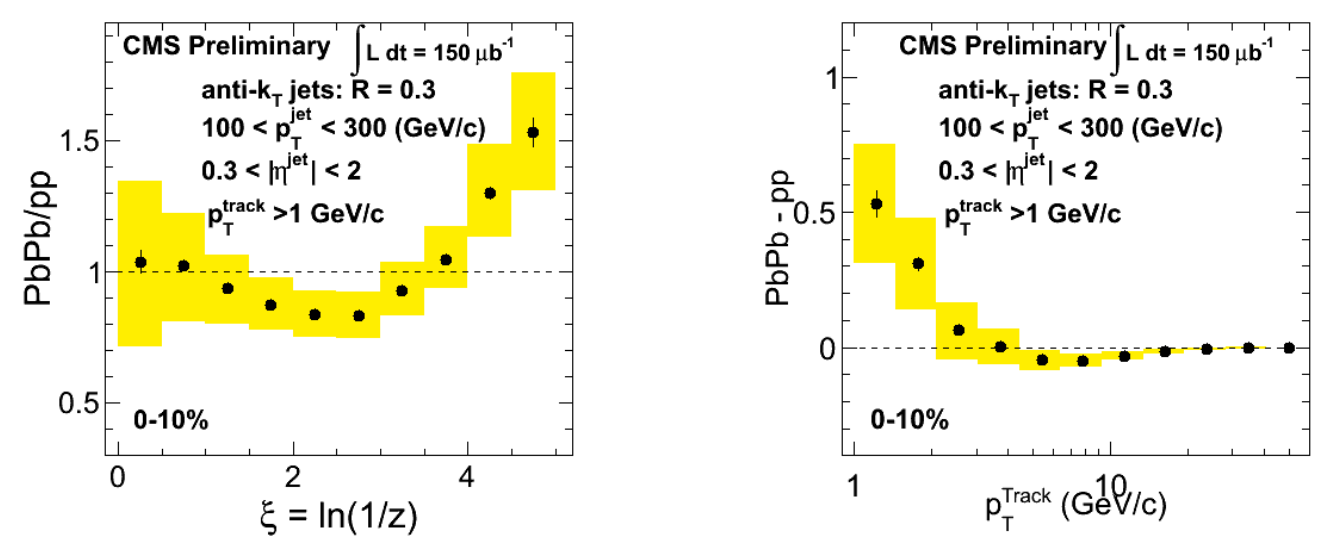

Figure 2. The ratio of jet fragmentation function in most central $\mathrm{PbPb}$ collisions to pp collisions for $p_{T}^{\text {jet }}>100 \mathrm{GeV} / c$ with track $p_{T}>1 \mathrm{GeV} / c$ as a function of $\xi$ (left) and track $p_{T}[11]$. 
located at the region $0.1<r<0.2$ and low $p_{T}$ particles are dominated at large radii, the depletion at intermediate radius indicating a suppression of intermediate $p_{T}$ particles is transported to larger radii with low $p_{T}$ particles enhancements by energy redistribution.

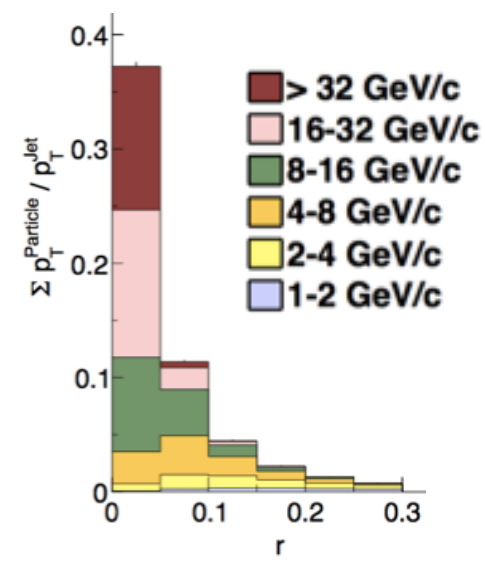

Figure 3. The distribution of charged particle fraction inside jet cones for various $p_{T}$ bins are shown as a function of the distance from jet axis.

\section{Summary}

The jet shapes and fragmentation functions measurements show consistent picture about the jet energy distribution inside the jet cone. The suppression of intermediate $p_{T}$ particles with low $p_{T}$ particles enhancements, indicate the energy is transported to large distance (low $p_{T}$ enhancement) from smaller jet radius (intermediate $p_{T}$ suppression). In summary, the jet quenching picture painted by CMS results has become more precise, detailed and quantitative, providing a new insight into the behavior of the high energy density medium formed in heavy-ion collisions.

\section{References}

[1] J. D. Bjorken, Energy loss of energetic partons in Quark-Gluon Plasma: Possible Extinction of High $p_{T}$ Jets in Hadron-Hadron Collisions, FERMILAB-PUB-82-059-THY.

URL http: //lss.fnal.gov/archive/1982/pub/Pub-82-059-T.pdf

[2] S. Chatrchyan, et al., Study of high- $p_{T}$ charged particle suppression in PbPb compared to $p p$ collisions at $\sqrt{s_{N N}}=2.76$ TeV, Eur. Phys. J. C 72 (2012) 1945. doi:10.1140/epjc/s 10052-012-1945-x.

[3] G. Aad, et al., Observation of a Centrality-Dependent Dijet Asymmetry in Lead-Lead Collisions at $\sqrt{s_{\mathrm{NN}}}=2.76$ TeV with the ATLAS Detector at the LHC, Phys. Rev. Lett. 105 (2010) 252303.

[4] K. Aamodt, et al., Suppression of Charged Particle Production at Large Transverse Momentum in Central Pb-Pb Collisions at $\sqrt{s_{N N}}=2.76$ TeV, Phys. Lett. B 696 (2011) 30. doi:10.1016/j.physletb.2010.12.020.

[5] CMS Collaboration, Very high-pT dihadron correlations in PbPb collisions at $\sqrt{s_{N N}}=2.76$ TeV, CMS-PAS-HIN-12-010. URL http: //cds . cern. ch/record/1472744

[6] CMS Collaboration, The CMS experiment at the CERN LHC, JINST s08004. doi:10.1088/1748-0221/3/08/S08004.

[7] S. Chatrchyan, et al., Measurement of jet fragmentation into charged particles in pp and PbPb collisions at $\sqrt{s_{N N}}=2.76$ TeV, JHEP 10 (2012) 087. arXiv:1205.5872, doi:10.1007/JHEP10(2012)087.

[8] I. Vitev, S. Wicks, B. W. Zhang, A theory of jet shapes and cross sections: from hadrons to nuclei, JHEP 11 (2008) 093. arXiv:0810.2807, doi:10.1088/1126-6708/2008/11/093.

[9] S. Chatrchyan, et al., Modification of jet shapes in PbPb collisions at $\sqrt{s_{N N}}=2.76 \mathrm{TeV}$, Phys. Lett. B 730 (2014) 243. URL http: //arxiv.org/abs/1310.0878

[10] S. Chatrchyan, et al., Observation and studies of jet quenching in $\mathrm{PbPb}$ collisions at nucleon-nucleon center-of-mass energy $=2.76 \mathrm{TeV}$, Phys. Rev. C 84 (2011) 024906. doi:10.1103/PhysRevC.84.024906.

[11] S. Chatrchyan, et al., Detailed Characterization of Jets in Heavy Ion Collisions Using Jet Shapes and Jet Fragmentation Functions, CMSPAS-HIN-12-013.

URL http://cds.cern.ch/record/1472734

[12] T. Sjöstrand, S. Mrenna, P. Skands, PYTHIA 6.4 physics and manual, JHEP 05 (2006) 026. arXiv:hep-ph/0603175, doi:10.1088/11266708/2006/05/026. 